\title{
Preparation and properties of radiation crosslinked natural rubber/clay nanocomposites
}

\begin{abstract}
Radiation crosslinking of natural rubber (NR)/clay nanocomposites was studied by using Standard Malaysian Rubber (SMRL) grade natural rubber and sodium montmorillonite (NaMMT) clay that had been modified with cationic surfactants, dodecyl ammonium chloride (DDA) and octadecylamine ammonium chloride (ODA). The NR/clay nanocomposites were prepared by melt mixing to produce NR/Na-MMT, NR/DDA-MMT and NR/ODA-MMT composites. X-ray diffraction results indicated the intercalation of the NR into silicate nanosize inter-layers for NR/DDA-MMT and NR/ODA-MMT nanocomposites. An optimum electron beam dose of $250 \mathrm{kGy}$ for crosslinking of NR, NR/DDA-MMT and NR/ODA-MMT was determined by gel content and tensile strength measurements. Upon electron beam irradiation, the tensile modulus of the nanocomposites keeps increasing with the increase of clay content up to $10 \mathrm{phr}$. However, the tensile strength and gel content of the nanocomposites show optimum values at a range of 3.0Ï $5.0 \mathrm{phr}$ clay content. From these results, NR/DDA-MMT shows higher tensile strength, whereas NR/ODA-MMT exhibits higher tensile modulus. The optimum tensile strengths of NR/DDA-MMT and NR/ODAMMT are 12.1 and 9.5 MPa, respectively. TGA studies showed that NR/DDA-MMT and NR/ODA-MMT nanocomposites have higher decomposition temperatures in comparison with the NR/Na-MMT.
\end{abstract}

Keyword: Natural rubber; Montmorillonite; Intercalate; Nanocomposites; Electron beam 